\section{Novel Signal Amplifica- tion Technology with Applications in DNA and Protein Detection Systems}

BioTechniques 30:1268-1272 (June 2001)

\section{ABSTRACT}

A non-enzymatic approach to signal am plification has practical advantages over conventional target amplification methods. We have designed a simple, cost-efficient signal amplification system that can be used to enhance the detection of nucleic acids or protein. The signal amplification process requires initial capture of analyte by a specific probe, which, depending on the analyte, can be an oligomer or an antibody. Once the analyte is captured, amplification moieties are applied to significantly enhance the sensitivity of analyte detection. Nucleic acid amplification is typically greater than 1000-fold, increasing the sensitivity of target detection to less than 1 amol/100 $\mu \mathrm{L}$. This amplification strategy presents a very flexible system with components that are easily altered to accommodate diverse assay requirements.

\section{INTRODUCTION}

Current nucleic acid detection methods employ enzyme-mediated target amplification strategies. Techniques such as PCR $(6,10)$, ligase chain reaction (12), and transcription-mediated amplification (11) are capable of producing millions of analyte copies, but not without limitation. Furthermore, many assays developed using these techniques require a secondary detection technology (3). A non-enzymatic approach that introduces multiple labels onto a target analyte for the downstream amplification of signal has many advantages over conventional target amplification methods (3). For instance, carryover contamination of amplified product, which must be controlled in target amplification systems to prevent false-positive results, becomes irrelevant when employing a system in which signal, not target, is being amplified. Sample-to-sample variation due to differences in amplification efficiency is minimized when the number of input target molecules is not altered (8). The most challenging problem associated with target amplification methods is the determination of target molecules in the sample before exponential amplification. With the use of appropriate internal standards, signal amplification is quantitative, as linearly amplified signal can be directly related to the number of molecules present in the starting sample $(1-3,14)$. Signal can be enhanced further to detect low copy number sequences with even greater sensitivity.

We have designed a novel signal am plification system that can be used to enhance the detection of nucleic acids or protein (5). The system requires analyte capture by a specific probe, which, depending on the analyte, can be an oligomer or an antibody. Once analyte is captured, our amplification molecule hybridizes to it. Multiple signaling moieties bind each amplification molecule to enhance detection. This two-step amplification system follows a simple protocol and can be adapted to an existing assay at minimal cost. Our unique system is comparable to commercially available signal amplification systems, most notably branched DNA (bDNA) and tyramide signal amplification (TSA). The bDNA assay (Bayer Diagnostics, Tarrytown, NY, USA) integrates a complex branched-DNA molecule into a unique hybridization assay to enhance signal $(1,2,8)$. Complex molecules and multiple hybridization steps make this a lengthy and laborious signal amplification system $(1,2,8)$. While the assay is highly sensitive $(1,3,7,8)$, its specific design limits the platforms to which it can be applied. NEN ${ }^{\circledR}$ Life Science Products (Boston, MA, USA) has designed a more versatile system, called $\mathrm{TS} \mathrm{A}^{\mathrm{TM}}$, that allows for multiple detection formats $(9,13,14)$. TSA is a quick and flexible assay that follows a relatively simple protocol; however, its level of sensitivity does not meet that of bDNA $(9,13,14)$. Our amplification method combines the attractive features of both TSA and bDNA to offer an innovative and sensitive amplification system that can be applied across multiple DNA and immunoassay formats. We present our approach to enhanced DNA detection through signal amplification.

\section{MATERIALS AND METHODS}

\section{PolyA Strand Synthesis}

The amplification molecule was synthesized on magnetic Streptavidin Dynabeads M-280 (Dynal, Oslo, Norway) coupled with biotinylated $\mathrm{dT}_{30}$ (Cortec DNA Service Laboratories, Kingston, ON, Canada). Briefly, $50 \mu \mathrm{L}$ (approximately $0.5 \mathrm{mg}$ ) of beads were magnetically separated and washed with $50 \mu \mathrm{L} 2 \times$ bind and wash buffer $(2 \times$ $\mathrm{B} \& \mathrm{~W}$ : $10 \mathrm{mM}$ Tris-HCl, pH 7.5, $1 \mathrm{mM}$ EDTA, $2 \mathrm{M} \mathrm{NaCl}$ ). Beads were then resuspended in $100 \mu \mathrm{L} 2 \times \mathrm{B} \& \mathrm{~W}$. Biotinylated-dT $30(400 \mathrm{pmol})$ diluted in $100 \mu \mathrm{L}$ distilled, deionized water were added to the $100 \mu \mathrm{L}$ bead suspension for $30 \mathrm{~min}$, at room temperature, with gentle rotation. Beads were separated and washed (as above) to remove uncoupled $\mathrm{dT}_{30}$, then resuspended in 100 $\mu \mathrm{L} 2 \times$ assay buffer I $(2 \mathrm{M} \mathrm{NaCl}, 200$ $\mathrm{mM}$ Tris, $0.16 \%$ Triton ${ }^{\circledR} \mathrm{X}-100, \mathrm{pH}$ 8.0). Poly(dA) (300 pmol) (Roche Molecular Biochemicals, Quebec, Canada) were diluted in $100 \mu \mathrm{L}$ distilled, deionized water and added to the bead suspension for a final volume of $200 \mu \mathrm{L}$ $(1 \times$ assay buffer I). Poly(dA) was hybridized to the coupled $\mathrm{dT}_{30}$ at $37^{\circ} \mathrm{C}$ for $60 \mathrm{~min}$, with gentle rotation. Beads were then separated and washed with $2 \times$ assay buffer I to remove any non-hybridized poly $(\mathrm{dA})$. Bound poly $(\mathrm{dA})$ molecules were then extended with terminal deoxynucleotidyl transferase (TdT; Amersham Pharmacia Biotech, Quebec, Canada). A $100-\mu \mathrm{L}$ reaction mixture consisting of $2 \mathrm{mM}$ dATP (Sigma-Aldrich, Oakville, ON, Canada), $1 \times$ OnePhorAll Plus (10 mM Tris-HCl, 10 $\mathrm{mM}$ magnesium acetate, $50 \mathrm{mM}$ potassium acetate), and $150 \mathrm{U}$ TdT was incubated with the beads overnight at $37^{\circ} \mathrm{C}$, with gentle rotation. Beads were separated and washed with OnePhorAll Plus and extended strands [herein referred to as PolyA] were eluted from the beads with $40 \mu \mathrm{L} 2 \mathrm{mM}$ EDTA, $\mathrm{pH}$ $8.0\left(65^{\circ} \mathrm{C}\right.$ for $\left.2 \mathrm{~min}\right)$. PolyA strands were visualized as a uniform band on a $0.8 \%$ agarose gel stained with methylene blue (Sigma-Aldrich). Length was determined against 0.24-9.5 kb RNA size ladder (Life Technologies, Burlington, ON, Canada), which was run simultaneously. 


\section{Assay Conditions}

To evaluate the amplification potential of our PolyA, we used a 96-well solid-phase DNA capture assay. The probe was a biotinylated 69 -mer hairpin oligonucleotide (4) consisting of a 16-bp duplex region and a 32-mer single-stranded capture region (Oligos Etc., Wilsonville, OR, USA). The synthetic target molecule used (Cortec DNA Service Laboratories) incorporated a $\mathrm{T}_{30}$-tail and single fluorescein isothiocyanate (FITC) label at its $5^{\prime}$ end, resulting in a labeled 62-mer target. PolyA strands were $18 \mathrm{~kb}$ in length and served as the amplification molecule. Oligo-dT 40 (Oligos Etc.) containing a single FITC label at each terminus provided the signaling moieties for the assay. All steps in the described protocol were carried out in a final reaction volume of $100 \mu \mathrm{L} / w e l l$.

Briefly, a 96-well Costar ${ }^{\mathrm{TM}}$ microplate (Fisher Scientific, Markham, ON, Canada) was coated with $20 \mathrm{pmol} /$ well avidin in $50 \mathrm{mM}$ carbonate buffer, $\mathrm{pH}$ 9.6 (Sigma-Aldrich). Biotinylated hairpin probe was diluted in $1 \times$ assay buffer I to $5 \mathrm{pmol} / 100 \mu \mathrm{L}$ and coupled to the avidin-coated plate for $30 \mathrm{~min}$ at $37^{\circ} \mathrm{C}$. Wells were washed six times with assay buffer I on an automated 96-well plate washer (Dynex, Chantilly, VA, USA). Serial dilutions of complementary target were prepared in $1 \times$ assay buffer I and hybridized to the probe for $1 \mathrm{~h}$ at $37^{\circ} \mathrm{C}$. Control wells received buffer in place of target. Wells were washed six times (as above). PolyA was diluted to $1 \times 10^{-4} \mathrm{pmol} / 100 \mu \mathrm{L}$ in blocking buffer (1 M NaCl, $100 \mathrm{mM}$ Tris, 0.08 $\%$ Triton X-100, 1\% BSA, pH 8.0), then added to bound target for $30 \mathrm{~min}$ at $37^{\circ} \mathrm{C}$. PolyA was not added to wells representing the non-amplified target. Wells were washed six times (as above). Oligo-dT 40 (Oligos Etc.) was diluted to $0.045 \mathrm{pmol} / 100 \mu \mathrm{L}$ blocking buffer $(450 \times$ molar excess signaling moiety to amplification molecule) and hybridized to the PolyA for $60 \mathrm{~min}$ at $37^{\circ} \mathrm{C}$. Wells were washed six times in assay buffer I. See schematic representation in Figure 1.

\section{Detection}

FITC labels were detected using a monoclonal anti-FITC-alkaline phos-

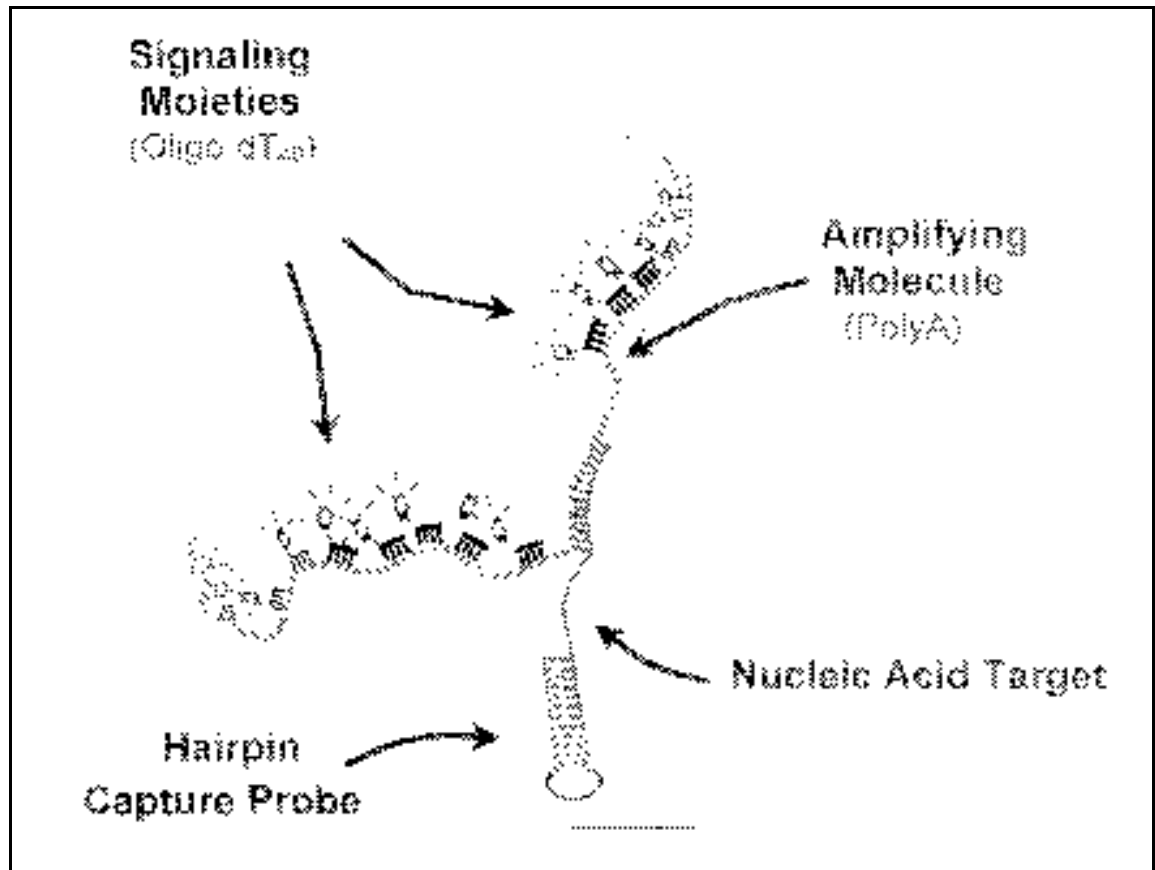

Figure 1. Schematic representation of our model DNA capture assay. A tethered DNA hairpin probe captures complementary target. The target's T-tail hybridizes a PolyA homopolymer, illustrated as the amplifying molecule. Short, labeled oligo-d $\mathrm{T}_{40}$ signaling moieties are subsequently hybridized along the molecule, and the signal is detected. Labeling of signal moieties will depend on the assay requirements and can be any of the standard labels for detection (fluorescent, chemiluminescent, isotopic, etc.). 


\section{Short Technical Reports}

phatase conjugate $(1.7 \mathrm{mg} / \mathrm{mL}$ stock; Sigma-Aldrich) diluted 2000-fold in 1× assay buffer II $(200 \mathrm{mM} \mathrm{NaCl}, 100$ 8.0). Wells were washed six times with assay buffer II and equilibrated for 5 min. CDP-Star ${ }^{\mathrm{TM}}$ substrate (Roche Molecular Biochemicals) was used to generate luminescent signal, which was monitored using an MLX luminometer (Dynex). Signal (S) was expressed as relative light units (RLU). Background (B) counts were derived from wells in which target was absent. Signal-tonoise ratio (S:N) was calculated as (S-B)/B, and an arbitrary value of 3 was set as an acceptable result. $\mathrm{mM}$ Tris, $0.08 \%$ Triton $\mathrm{X}-100, \mathrm{pH}$

\section{RESULTS AND DISCUSSION}

Our amplification system enhanced the sensitivity of our genotyping assay by greater than 1000 -fold over the same assay performed using target labeled directly with a single FITC moiety (Figure 2). The highest sensitivity achieved without amplification fell between 1000 and $10000 \mathrm{amol} / 100 \mu \mathrm{L}$. Although 1000 amol/100 $\mu \mathrm{L}$ was clearly detected, it did not meet our arbitrary $\mathrm{S}: \mathrm{N}$ threshold of 3 . Where signal amplification was applied, sensitivity increased to less than $1 \mathrm{amol} / 100 \mu \mathrm{L}$ of detectable target with a $\mathrm{S}: \mathrm{N}$ ratio greater than 3 (Figure 2B). Although
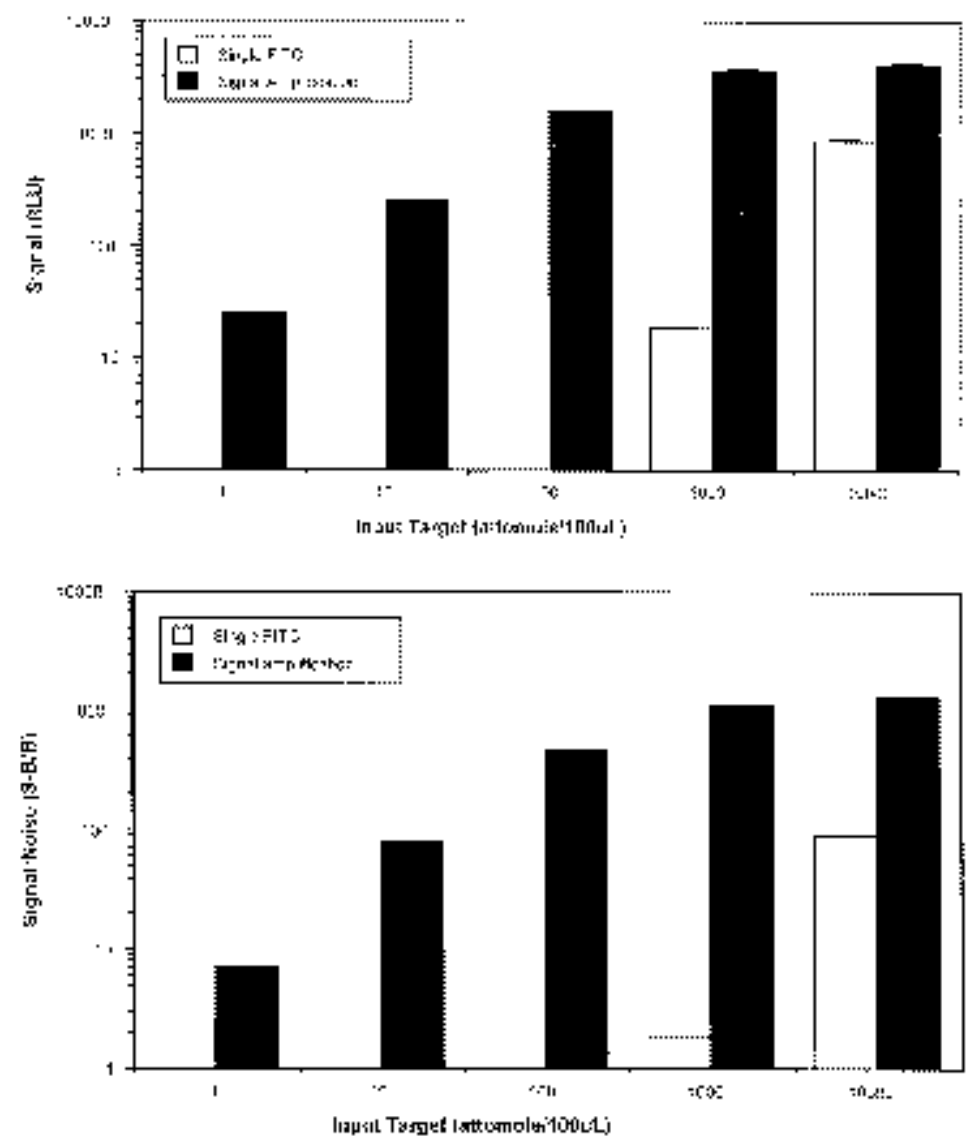

Figure 2. Comparison of signal amplified target with directly labeled target. Data were generated using our model genotyping assay (see text). Serial dilutions (1-10000 amol) of complementary target were added to plates coupled with DNA hairpin probes. Half of the plate was subjected to signal amplification, while the other half was not. (A) Absolute signals, expressed as RLU, are represented for each target dilution with error bars. The white bars represent detection of target labeled with a single FITC moiety. The solid bars represent amplified signal. Application of our amplification system resulted in at least a 1000-fold increase in the level of detectable target. (B) Sensitivity of target detection increased from greater than $1000 \mathrm{amol} / 100 \mu \mathrm{L}$ to less than $1 \mathrm{amol} / 100 \mu \mathrm{L}$ with our signal amplification. An arbitrary S:N value of 3 was set to denote significant signals above background. the dynamic range of this assay was less than 3 logs, we do not view this as a limitation of the system. Dynamic range depends on length of the PolyA and oligo(dT) molecules used, as well as the number of signaling moieties to be detected, and thus can be increased by varying such parameters.

This amplification strategy presents a very flexible system with components that are easily altered to accommodate diverse assay requirements. For the purpose of our model system, the target has been synthetically modified to contain a T-tail for PolyA capture; however, alternative methods for addition of T-tails exist such as oligo(dT) tailing using terminal transferase or through biotin/streptavidin interactions. For various immunoassay and protein detection systems, antibody may be crosslinked to oligo(dTs), providing a substrate for PolyA hybridization. In instances when target modification is not desirable, a specific T-tailed intermediate oligonucleotide may be hybridized to the already bound target, followed by addition of amplification and signaling molecules, as described.

The homogeneity of PolyA strands being synthesized was assessed with methylene blue staining. Synthesis generated a homogeneous strand population (data not shown), thus indicating that the protocol is highly reproducible under the described conditions. Moreover, individual components of the PolyA extension reaction (dATP, TdT, extension time, etc.) may be adjusted to generate molecules of various lengths and nucleotide composition (data not shown). Additionally, modified nucleotides may be directly incorporated into PolyA, eliminating the need for subsequent oligo(dT) hybridization. This may prove especially useful as novel nucleotide conjugates become available. We have successfully integrated FITC-, digoxigenin (DIG)-, amino-, and biotin-modified nucleotides into our PolyA molecule (data not shown).

In summary, we have developed a robust signal amplification method that significantly improves the sensitivity of analyte detection across a number of assay formats. Our unique amplification molecule generates a substantial increase in an assay's sensitivity without the problems associated with target 
amplification methods. The protocol can be directly applied to several types of assays including single nucleotide polymorphism (SNP) analysis, clinical diagnostics for pathogen detection, and gene expression profiling where microarray technology is in current use. The adaptability of this technology to multiple existing assay formats provides a seamless transition to enhanced assay performance.

\section{REFERENCES}

1.Christopoulos, T.K. 1999. Nucleic acid analysis. Anal. Chem. 71:425-438.

2.Collins, M., B. Irvine, D. Tyner, E. Fine, C. Zayati, C. Chang, T. Horn, D. Ahle et al. 1997. A branched DNA signal amplification assay for quantification of nucleic acid targets below 100 molecules/mL. Nucleic Acids Res. 25:2979-2984.

3.Kricka, L.J. 1999. Nucleic acid detection technologies-labels, strategies, and formats. Clin. Chem. 45:453-458.

4.Lane, M.J., A.S. Benight, and B.D. Faldasz. Nucleic Acid Capture Moieties. US Patent 5770365. June 23, 1998.

5.Lane, M.J., A.S. Benight, and B.D. Faldasz. Signal amplification method. US Patent 5902724. May 11, 1999.

6.Mullis, K.B. and F.A. Faloona. 1987. Specific synthesis of DNA in vitro via a polymerasecatalyzed chain reaction. Methods Enzymol. 155:335-350.

7.Pawlotsky, J., A. Bastie, I. Lonjon, J. Rémiré, F. Darthuy, C. Soussy, and D. Dhumeaux. 1997. What technique should be used for routine detection and quantification of HBV DNA in clinical samples? J. Virol. Methods 65:245-253.

8.Nolte, F. 1998. Branched DNA signal amplification for direct quantitation of nucleic acid sequences in clinical specimens. Adv. Clin. Chem. 33:201-217.

9.Roth, K.A., K. Adler, and M.N. Bobrow. 1999. Enhanced tyramide signal amplification immunohistochemical detection. J. Histochem. Cytochem. 47:1644-1645.

10.Saiki, R.K., T. Scharf, F. Faloona, K.B. Mullis, G.T. Horn, H.A. Erlich, and N. Arnheim. 1985. Enzymatic amplification of bglobin genomic sequences and restriction site analysis for diagnosis of sickle cell anemia. Science 230:1350-1354.

11.Simpkins, S.A., A.B. Chan, J. Hays, B. Pöpping, and N. Cook. 2000. An RNA transcription-based amplification technique (NASBA) for the detection of viable Salmonella enterica. Lett. Appl. Microbiol. 30:75-79.

12.Wu, D.Y. and R.B. Wallace. 1989. The ligation amplification reaction (LAR) - amplification of specific DNA sequences using sequential rounds of template-dependent ligation. Genomics 4:560-569.

13. Yang, H., I.B. Wanner, S.D. Roper, and N. Chaudhari. 1999. An optimized method for in situ hybridization with signal amplification that allows the detection of rare mRNAs. J. Histochem. Cytochem. 47:431-445.

14.Zerbini, M., M. Cricca, G. Gentilomi, S. Venturoli, G. Gallinella and M. Musiani. 2000. Tyramide signal amplification of biotinylated probe in dot-blot hybridization assay for the detection of parvovirus B19 DNA in serum samples. Clin. Chim. Acta 307:79-87.

Address correspondence to Connie Lisle, Tm Bioscience Corporation, 439 University Avenue, Suite 1100, Toronto, ON M5G 1Y8, Canada. e-mail: conniem@ tmbioscience.com

Received 30 June 2000; accepted 20 December 2000.

C.M. Lisle, S. Bortolin, A.S. Benight $^{1}$, R.A. Janeczko, and R.L. Zastawny

Tm Bioscience Corporation

Toronto, ON, Canada

${ }^{1}$ University of Illinois

Chicago, IL, USA

\section{Fluorescent Staining of Glycoproteins on Poly- vinylidene Difluoride Membrane with 8-Aminonaphthalene- 1,3,6-Trisulfonate}

BioTechniques 30:1272-1278 (June 2001)

\section{ABSTRACT}

Here, we describe a simple and sensitive method that allows fluorescent detection of glycoproteins on polyvinylidene difluoride $(P V D F)$ membrane. We used periodic acid oxidation of carbohydrate chains of glycoproteins and fluorescent labeling with 8 aminonaphthalene-1,3,6-trisulfonate (AN $T S)$ by reductive amination. We developed an additional method to enhance the ability of PVDF to absorb glycoproteins by using non-glycoprotein lectin, such as wheat germ agglutinin (WGA), as a link between the PVDF membrane and glycoproteins, resulting in considerably increased detection sensitivity to glycoproteins.

\section{INTRODUCTION}

Protein glycosylation, a universal but complicated co- and post-translational modification of proteins in eukaryote cells (26), has been found to be very important to glycoprotein and many other biological processes $(4,7$, $22,23,26)$. To comprehensively understand a sophisticated biological system, researchers often determine if a preparation contains glycoproteins or if a protein is glycosylated. For this reason, many staining methods have been developed to detect glycoproteins $(1,2$, $5,8-14,17,18,20,21,24,27,28)$. These methods can be roughly classified into three general categories. One category is lectin blotting based on specific and noncovalent lectin binding to oligosaccharides of protein $(11,12)$. Lectins are pre-biotinylated or avidin conjugated with horseradish peroxidase or alkaline phosphatase $(13,17,20,27)$. The resulting complexes are then revealed either by a chemiluminescent or a colorimetric reaction. Alternatively, lectins have 